\title{
The noncommutative sine-Gordon breather
}

Cite as: J. Math. Phys. 50, 095201 (2009); https://doi.org/10.1063/1.3093825

Submitted: 17 November 2008 . Accepted: 23 January 2009 . Published Online: 09 April 2009

André Fischer, and Olaf Lechtenfeld

\section{Where in the world is AIP Publishing?}

Find out where we are exhibiting next 


\title{
The noncommutative sine-Gordon breather
}

\author{
André Fischer ${ }^{\mathrm{a})}$ and Olaf Lechtenfeld ${ }^{\mathrm{a})}$ \\ Institut für Theoretische Physik, Leibniz Universität Hannover, Appelstraße 2, 30167 \\ Hannover, Germany
}

(Received 17 November 2008; accepted 23 January 2009; published online 9 April 2009)

\begin{abstract}
As shown by Lechtenfeld et al. [Nucl. Phys. B 705, 447 (2005)], there exists a noncommutative deformation of the sine-Gordon model which remains (classically) integrable but features a second scalar field. We employ the dressing method (adapted to the Moyal-deformed situation) for constructing the deformed kinkantikink and breather configurations. Explicit results and plots are presented for the leading noncommutativity correction to the breather. Its temporal periodicity is unchanged. (C) 2009 American Institute of Physics. [DOI: 10.1063/1.3093825]
\end{abstract}

\section{INTRODUCTION AND SUMMARY}

The sine-Gordon model is a paradigm for relativistic integrable models in $1+1$ dimensions (e.g., see Ref. 1). Its multisoliton spectrum is well known and consists not only of multikink scattering configurations but also of bound states, the simplest of which is the so-called breather. It may be obtained formally by analytically continuing the kink-antikink configuration in its relative velocity variable, $\mathrm{v} \rightarrow i \mathrm{v}$, and oscillates periodically in time.

A systematic procedure for deriving the integrability features of the sine-Gordon model relates it to the self-duality equations of SU(2) Yang-Mills theory in $2+2$ dimensions. $^{2}$ In a light-cone gauge, these equations follow from the Nair-Schiff action. ${ }^{3}$ A first and straightforward dimensional reduction produces Ward's modified chiral sigma-model action for SU(2)-valued fields in $2+1$ dimensions. ${ }^{4}$ A second dimensional reduction then generates an Abelian sigma model equivalent to the sine-Gordon theory. To arrive there, one must prescribe a particular dependence on one spatial coordinate (rather than trivial independence) and also algebraically restrict the field from $\mathrm{SU}(2)$ to a U(1) subgroup. The remaining phase $\varphi(t, x)$ turns out to be ruled by the sine-Gordon equation, with the coupling or mass $\alpha$ appearing as a parameter of the dimensional reduction.

For several years now the Moyal deformation of integrable field theories has been of some interest. In particular, the Ward model ${ }^{5-7}$ and the sine-Gordon model ${ }^{8,9}$ have been generalized to the noncommutative realm. The key insight for the latter case was that the extension of SU(2) to $\mathrm{U}(2)$, necessary for implementing the Moyal deformation in the Yang-Mills theory, should be retained under the dimensional and algebraic reduction, so that the noncommutative sigma-model field takes its values in $\mathrm{U}(1) \times \mathrm{U}(1)$ rather than $\mathrm{U}(1)$. The deformed sine-Gordon model so obtained $^{8}$ features two scalar fields (phases) $\phi_{+}$and $\phi_{-}$, whose noncommutative Abelian WessZumino-Witten (WZW) actions are coupled in a simple way. In the commutative limit, the average $\varphi=\frac{1}{2}\left(\phi_{+}+\phi_{-}\right)$of these phases produces the standard sine-Gordon field while their difference $\phi_{+}$ $-\phi_{-}$decouples as a free field.

Since the powerful techniques for constructing multisoliton solutions in integrable models have been shown to survive the noncommutative deformation, it is straightforward (but may be tedious) to work out such configurations for the Moyal-deformed sine-Gordon model as well. The basic strategy was already outlined in Ref. 5 but has been applied only to the simple kink so far. ${ }^{8}$ However, owing to the relativistic invariance, a one-kink configuration depends only on its single

a)Electronic addresses: afischer@itp.uni-hannover.de and lechtenf@itp.uni-hannover.de. 
comoving coordinate $\eta(t, x)$, and so it cannot get deformed. Only multilumps with relative motion should be affected by noncommutativity. The first instances are the two-kink, kink-antikink, and breather solutions.

In this letter we apply the Moyal deformation to the two latter cases. It is important to verify the effect of noncommutativity, since the tree-level computations of Ref. 8 had suggested that perhaps the entire Moyal deformation of the sine-Gordon model might be ficticious. Here, we demonstrate this not to be the case, by working out the first-order (in the noncommutativity parameter) correction to the "classical" kink-antikink and breather configurations. It turns out that this leading correction affects only the would-be free field $\phi_{+}-\phi_{-}$; the generalized sine-Gordon field $\varphi$ gets modified at second order onward, as does the energy density. Only the substantial calculational effort prevented us from evaluating higher orders, but we present the starting-point equations for doing so. As an exact result, the temporal periodicity of the breather is unchanged by the deformation.

\section{THE MODEL}

The integrable noncommutative sine-Gordon model introduced in Ref. 8 involves two U(1)valued fields,

$$
g_{+}(t, x)=e_{\star}^{\frac{i}{2} \phi_{+}(t, x)} \in \mathrm{U}(1)_{+} \quad \text { and } \quad g_{-}(t, x)=e_{\star}^{-\frac{i}{2} \phi_{-}(t, x)} \in \mathrm{U}(1)_{-},
$$

and may be defined via its action,

$$
S\left[g_{+}, g_{-}\right]=S_{\mathrm{WZW}}\left[g_{+}\right]+S_{\mathrm{WZW}}\left[g_{-}\right]+\alpha^{2} \int d t d x\left(g_{+}^{\dagger} g_{-}+g_{-}^{\dagger} g_{+}-2\right),
$$

where $S_{\mathrm{WZW}}$ is the Abelian WZW action

$$
S_{\mathrm{WZW}}[g]=-\frac{1}{2} \int d t d x\left(\partial_{t} g^{\dagger} \partial_{t} g-\partial_{x} g^{\dagger} \partial_{x} g\right)-\int d t d x \int_{0}^{1} d \lambda \hat{g}^{\dagger} \partial_{[t} \hat{g} \star \hat{g}^{\dagger} \partial_{x]} \hat{g} \star \hat{g}^{\dagger} \partial_{\lambda} \hat{g},
$$

with a homotopy path $\hat{g}(\lambda)$ connecting $\hat{g}(0)=1$ and $\hat{g}(1)=g$ and a Moyal star product

$$
\left(f_{1} \star f_{2}\right)(t, x)=f_{1}(t, x) \exp \left\{\frac{i}{2}\left(\overleftarrow{\partial}_{t} \theta \vec{\partial}_{x}-\overleftarrow{\partial}_{x} \theta \vec{\partial}_{t}\right\} f_{2}(t, x) \quad \text { so that } \quad[t, x]_{\star}=i \theta\right.
$$

In light-cone variables,

$$
u:=\frac{1}{2}(t+x), \quad v:=\frac{1}{2}(t-x), \quad \partial_{u}=\partial_{t}+\partial_{x}, \quad \partial_{v}=\partial_{t}-\partial_{x},
$$

the corresponding equations of motion read

$$
\begin{gathered}
\partial_{v}\left(g_{+}^{\dagger} \star \partial_{u} g_{+}+g_{-}^{\dagger} \star \partial_{u} g_{-}\right)=0, \\
\partial_{v}\left(g_{+}^{\dagger} \star \partial_{u} g_{+}-g_{-}^{\dagger} \star \partial_{u} g_{-}\right)=2 \alpha^{2}\left(g_{+}^{\dagger} \star g_{-}-g_{-}^{\dagger} \star g_{+}\right),
\end{gathered}
$$

which in the commutative limit $\theta \rightarrow 0$ simplifies to

$$
\partial_{u} \partial_{v}\left(\phi_{+}-\phi_{-}\right)=0 \text { and } \partial_{u} \partial_{v}\left(\phi_{+}+\phi_{-}\right)=-8 \alpha^{2} \sin \frac{1}{2}\left(\phi_{+}+\phi_{-}\right) .
$$

Hence, the identification of the standard sine-Gordon field $\varphi$ with mass $2 \alpha$ is made via

$$
\frac{1}{2}\left(\phi_{+}+\phi_{-}\right)=\varphi+O(\theta) \text { or } \quad g_{-}^{\dagger} \star g_{+}=e^{i \varphi}+O(\theta) .
$$

For later use, we embed the U(1)-valued fields into $\mathrm{U}(2)$, 


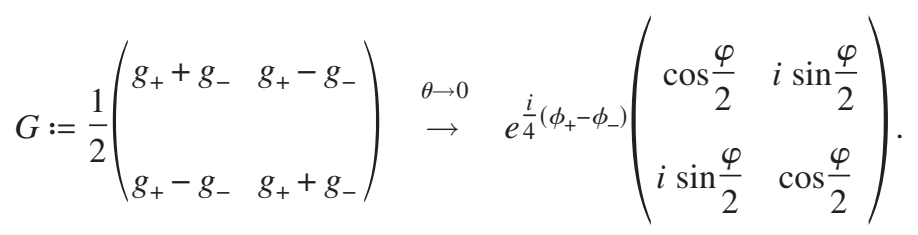

\section{DRESSING CONSTRUCTION}

The breather solution may be obtained from a kink-antikink configuration with relative velocity $2 \mathrm{v}$ by an analytic continuation $\mathrm{v} \rightarrow i \mathrm{v}$. The comoving coordinates for the kink and antikink read

$$
\eta_{1}=+p u-\frac{1}{p} v=+\gamma(x-\mathrm{v} t) \quad \text { and } \quad \eta_{2}=-\frac{1}{p} u+p v=-\gamma(x+\mathrm{v} t)
$$

respectively, where $p \in(0,1)$,

$$
\mathrm{v}=\frac{1-p^{2}}{1+p^{2}}>0 \Leftrightarrow p^{2}=\frac{1-\mathrm{v}}{1+\mathrm{v}} \quad \text { and } \quad \gamma=\frac{1}{\sqrt{1-\mathrm{v}^{2}}}=\frac{1}{2}\left(p+\frac{1}{p}\right) .
$$

A convenient way to construct the kink-antikink solution employs the dressing method. For the case at hand, it yields 5

$$
\begin{aligned}
G= & 1-2\left(1+\frac{1-\mathrm{v}}{\mathrm{v}} P_{2}\right) \star T_{1} \star\left[T_{1}^{\dagger} \star\left(1-\sigma P_{2}\right) \star T_{1}\right]_{\star}^{-1} \star T_{1}^{\dagger} \\
& -2\left(1-\frac{1+\mathrm{v}}{\mathrm{v}} P_{1}\right) \star T_{2} \star\left[T_{2}^{\dagger} \star\left(1-\sigma P_{1}\right) \star T_{2}\right]_{\star}^{-1} \star T_{2}^{\dagger} \\
= & 1-2\left(1+\frac{1-\mathrm{v}}{\mathrm{v}} P_{2}\right) \star P_{1} \star\left[1-\sigma P_{2} \star P_{1}\right]_{\star}^{-1} \\
& -2\left(1-\frac{1+\mathrm{v}}{\mathrm{v}} P_{1}\right) \star P_{2} \star\left[1-\sigma P_{1} \star P_{2}\right]_{\star}^{-1}
\end{aligned}
$$

where $\sigma=-4 p^{2} /\left(1-p^{2}\right)^{2}=1-\mathrm{v}^{-2}$, and we introduced Hermitian projectors

$$
P_{1}=T_{1} \star\left(T_{1}^{\dagger} \star T_{1}\right)_{\star}^{-1} \star T_{1}^{\dagger} \quad \text { and } P_{2}=T_{2} \star\left(T_{2}^{\dagger} \star T_{2}\right)_{\star}^{-1} \star T_{2}^{\dagger}
$$

based on $2 \times 1$ matrix-valued functions $T_{1}\left(\eta_{1}\right)$ and $T_{2}\left(\eta_{2}\right)$ related to the kink and antikink components of the configuration. The $T_{i}$ are determined only up to right (star) multiplication with an arbitrary invertible function and may be taken as

$$
T_{1}=\left(\begin{array}{c}
1 \\
i e^{2 \alpha \eta_{1}}
\end{array}\right) \text { and } T_{2}=\left(\begin{array}{c}
1 \\
-i e^{2 \alpha \eta_{2}}
\end{array}\right)
$$

by a suitable choice of the coordinate origin. Note that we have dropped the star index on the exponentials since each one depends on a single coordinate combination only.

By inserting these $T_{i}$ into (3.4), the ensuing projectors into (3.3), one is in principle able to read off $g_{ \pm}$from (2.9) and extract the noncommutative breather configuration $\phi_{ \pm}$.

\section{COMMUTATIVE BREATHER}

Before delving into the explicit computation, let us first retrieve the familiar commutative breather in the $\theta \rightarrow 0$ limit.

Since a coordinate rescaling modifies the coupling $\alpha$, we take the freedom to put $2 \alpha=1$ in the following. Dropping all stars, one first builds 


$$
P_{1}=\frac{1}{1+e^{2 \eta_{1}}}\left(\begin{array}{cc}
1 & -i e^{\eta_{1}} \\
i e^{\eta_{1}} & e^{2 \eta_{1}}
\end{array}\right) \text { and } P_{2}=\frac{1}{1+e^{2 \eta_{2}}}\left(\begin{array}{cc}
1 & i e^{\eta_{2}} \\
-i e^{\eta_{2}} & e^{2 \eta_{2}}
\end{array}\right)
$$

and thus

$$
\left[T_{j}^{\dagger}\left(1-\sigma P_{k}\right) T_{j}\right]^{-1}=\frac{1+e^{2 \eta_{k}}}{(1-\sigma)\left(1-e^{\eta_{1}+\eta_{2}}\right)^{2}+\left(e^{\eta_{1}}+e^{\eta_{2}}\right)^{2}} \quad \text { for }(j, k)=(1,2) \quad \text { or }
$$

Next, one obtains

$$
\begin{aligned}
& \left(1+\frac{1-\mathrm{v}}{\mathrm{v}} P_{2}\right) T_{1} T_{1}^{\dagger}=\frac{1}{1+e^{2 \eta_{2}}}\left(\begin{array}{cc}
\frac{1}{\mathrm{v}}+e^{2 \eta_{2}} & i \frac{1-\mathrm{v}}{\mathrm{v}} e^{\eta_{2}} \\
-i \frac{1-\mathrm{v}}{\mathrm{v}} e^{\eta_{2}} & 1+\frac{1}{\mathrm{v}} e^{2 \eta_{2}}
\end{array}\right)\left(\begin{array}{cc}
1 & -i e^{\eta_{1}} \\
i e^{\eta_{1}} & e^{2 \eta_{1}}
\end{array}\right), \\
& \left(1-\frac{1+\mathrm{v}}{\mathrm{v}} P_{1}\right) T_{2} T_{2}^{\dagger}=\frac{1}{1+e^{2 \eta_{1}}}\left(\begin{array}{cc}
-\frac{1}{\mathrm{v}}+e^{2 \eta_{1}} & i \frac{1+\mathrm{v}}{\mathrm{v}} e^{\eta_{1}} \\
-i \frac{1+\mathrm{v}}{\mathrm{v}} e^{\eta_{1}} & 1-\frac{1}{\mathrm{v}} e^{2 \eta_{1}}
\end{array}\right)\left(\begin{array}{cc}
1 & i e^{\eta_{2}} \\
-i e^{\eta_{2}} & e^{2 \eta_{2}}
\end{array}\right),
\end{aligned}
$$

which combine to

$$
\begin{aligned}
G & =\frac{1}{\mathrm{v}^{-2}\left(1-e^{\eta_{1}+\eta_{2}}\right)^{2}+\left(e^{\eta_{1}}+e^{\eta_{2}}\right)^{2}}\left(\begin{array}{cc}
\mathrm{v}^{-2}\left(1-e^{\eta_{1}+\eta_{2}}\right)^{2}-\left(e^{\eta_{1}}+e^{\eta_{2}}\right)^{2} & 2 i \mathrm{v}^{-1}\left(e^{\eta_{1}}+e^{\eta_{2}}\right)\left(1-e^{\eta_{1}+\eta_{2}}\right) \\
2 i \mathrm{v}^{-1}\left(e^{\eta_{1}}+e^{\eta_{2}}\right)\left(1-e^{\eta_{1}+\eta_{2}}\right) & \mathrm{v}^{-2}\left(1-e^{\eta_{1}+\eta_{2}}\right)^{2}-\left(e^{\eta_{1}}+e^{\eta_{2}}\right)^{2}
\end{array}\right) \\
& =\frac{1}{\sinh ^{2} \gamma \mathrm{v} t+\mathrm{v}^{2} \cosh ^{2} \gamma x}\left(\begin{array}{cc}
\sinh ^{2} \gamma \mathrm{v} t-\mathrm{v}^{2} \cosh ^{2} \gamma x & 2 i \mathrm{v} \sinh \gamma \mathrm{v} t \cosh \gamma x \\
2 i \mathrm{v} \sinh \gamma \mathrm{v} t \cosh \gamma x & \sinh ^{2} \gamma \mathrm{v} t-\mathrm{v}^{2} \cosh ^{2} \gamma
\end{array}\right),
\end{aligned}
$$

with the help of

$$
\eta_{1}+\eta_{2}=-2 \gamma \mathrm{v} t \text { and } \quad \eta_{1}-\eta_{2}=2 \gamma x
$$

Comparing with (2.9) we learn that, with $\phi_{+}-\phi_{-}=: 4 \beta$,

$$
e^{i \beta} \cos \frac{\varphi}{2}=\frac{\sinh ^{2} \gamma \mathrm{v} t-\mathrm{v}^{2} \cosh ^{2} \gamma x}{\sinh ^{2} \gamma \mathrm{v} t+\mathrm{v}^{2} \cosh ^{2} \gamma x} \quad \text { and } \quad e^{i \beta} \sin \frac{\varphi}{2}=\frac{2 \mathrm{v} \sinh \gamma \mathrm{v} t \cosh \gamma x}{\sinh ^{2} \gamma \mathrm{v} t+\mathrm{v}^{2} \cosh ^{2} \gamma x}
$$

so that

$$
\tan \frac{\varphi}{4} \equiv \frac{\sin \frac{\varphi}{2}}{1+\cos \frac{\varphi}{2}}=\frac{2 \mathrm{v} \sinh \gamma \mathrm{v} t \cosh \gamma x}{\left(e^{i \beta}+1\right) \sinh ^{2} \gamma \mathrm{v} t+\left(e^{i \beta}-1\right) \mathrm{v}^{2} \cosh ^{2} \gamma x}
$$

analytically continues via $\mathrm{v} \rightarrow i \mathrm{v}$ to

$$
\tan \frac{\bar{\varphi}}{4}=\frac{2 \mathrm{v} \sin \bar{\gamma} \mathrm{v} t \cosh \bar{\gamma} x}{\left(e^{i \beta}+1\right) \sin ^{2} \bar{\gamma} \mathrm{v} t+\left(e^{i \beta}-1\right) \mathrm{v}^{2} \cosh ^{2} \bar{\gamma} x},
$$

with $\bar{\gamma}=1 / \sqrt{1+\mathrm{v}^{2}}$. Since $\bar{\varphi}$ is real we must have $\beta=0$ or $\beta=\pi$. The boundary condition $\bar{\varphi} \rightarrow 0$ for $|x| \rightarrow \infty$ selects the second option, ${ }^{1}$ and we have recovered the celebrated breather configuration ${ }^{1}$

${ }^{1}$ Alternatively, begin with $\phi_{+}=\phi_{-}=\varphi$ and shift $\phi_{ \pm} \rightarrow \phi_{ \pm} \pm 2 \pi$, or else, put $\beta=0$ and shift $\varphi \rightarrow \varphi+2 \pi$. 


$$
-\bar{\varphi}=4 \arctan \left\{\frac{\sin \bar{\gamma} v t}{\mathrm{v} \cosh \bar{\gamma} x}\right\} .
$$

\section{NONCOMMUTATIVE CONSTRUCTION}

When attempting to repeat the above computation in the Moyal-deformed case, one must account for the noncommutativity of the comoving coordinates,

$$
[t, x]_{\star}=i \theta \Rightarrow\left[\eta_{1}, \eta_{2}\right]_{\star}=2 i \theta \gamma^{2} \mathrm{v}=2 i \theta \frac{\mathrm{v}}{1-\mathrm{v}^{2}}=: i \lambda,
$$

which leads to the fundamental intertwining relation,

$$
e^{\left(a_{1}+b_{1}\right) \eta_{1}+\left(a_{2}+b_{2}\right) \eta_{2}}=e^{-(i / 2) \lambda a \wedge b} e^{a_{1} \eta_{1}+a_{2} \eta_{2}} \star e^{b_{1} \eta_{1}+b_{2} \eta_{2}}=e^{(1 / 2)\left(a_{1} \eta_{1}+a_{2} \eta_{2}\right)} \star e^{b_{1} \eta_{1}+b_{2} \eta_{2}} \star e^{(1 / 2)\left(a_{1} \eta_{1}+a_{2} \eta_{2}\right)},
$$

which (for $f$ regular at zero) implies

$$
e^{a_{1} \eta_{1}+a_{2} \eta_{2}} \star f\left(e^{b_{1} \eta_{1}+b_{2} \eta_{2}}\right)=f\left(e^{b_{1} \eta_{1}+b_{2} \eta_{2}+i \lambda a \wedge b}\right) \star e^{a_{1} \eta_{1}+a_{2} \eta_{2}} .
$$

Again, we put $2 \alpha=1$ for convenience. The projectors (4.1) are unaffected by the deformation, but the star products become relevant when $T_{1}$ or $P_{1}$ meets $T_{2}$ or $P_{2}$. As a basic ingredient in (3.3), we first compute

$$
\begin{aligned}
& T_{j}^{\dagger} \star\left(1-\sigma P_{k}\right) \star T_{j} \\
& =\left(1,-i e^{\eta_{j}}\right) \star\left[\left(1+e^{\left.2 \eta_{k}\right)^{-(1 / 2)}}\left(\begin{array}{cc}
1-\sigma+e^{2 \eta_{k}} & -i \sigma e^{\eta_{k}} \\
i \sigma e^{\eta_{k}} & 1+(1-\sigma) e^{2 \eta_{k}}
\end{array}\right)\left(1+e^{\left.2 \eta_{k}\right)^{-(1 / 2)}}\right] \star\left(\begin{array}{c}
1 \\
i e^{\eta_{j}}
\end{array}\right)\right.\right. \\
& =\left(1+e^{2 \eta_{k}}\right)^{-(1 / 2)} \star\left(1-\sigma+e^{2 \eta_{k}}\right) \star\left(1+e^{2 \eta_{k}}\right)^{-(1 / 2)}
\end{aligned}
$$

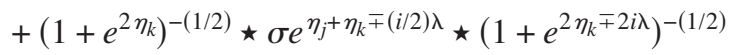

$$
\begin{aligned}
& +\left(1+e^{2 \eta_{k} \pm 2 i \lambda}\right)^{-(1 / 2)} \star \sigma e^{\eta_{j}+\eta_{k} \pm(i / 2) \lambda} \star\left(1+e^{\left.2 \eta_{k}\right)^{-(1 / 2)}}\right. \\
& +\left(1+e^{2 \eta_{k} \pm 2 i \lambda}\right)^{-(1 / 2)} \star\left(e^{\eta_{j}}+(1-\sigma) e^{2 \eta_{j}+2 \eta_{k}}\right) \star\left(1+e^{2 \eta_{k} \mp 2 i \lambda}\right)^{-(1 / 2)} \\
& =\left(1+e^{2 \eta_{k}}\right)^{-(1 / 2)} \star\left[(1-\sigma)\left(1-e^{\eta_{1}+\eta_{2}}\right)^{2}+\left(e^{\eta_{1}}+e^{\eta_{2}}\right)^{2}\right] \star\left(1+e^{2 \eta_{k}}\right)^{-(1 / 2)}+O\left(\lambda^{2}\right) .
\end{aligned}
$$

In the last step, we dropped terms of $O\left(\lambda^{2}\right)$ in order to arrive at a manageable expression. Inserting the above into (3.3) and abbreviating

$$
N_{k}=T_{k}^{\dagger} T_{k}=1+e^{2 \eta_{k}} \quad \text { and } \quad D=(1-\sigma)\left(1-e^{\eta_{1}+\eta_{2}}\right)^{2}+\left(e^{\eta_{1}}+e^{\eta_{2}}\right)^{2},
$$

we find the matrix elements of $G$ up to $O\left(\lambda^{2}\right)$ (denoted by “ $\simeq$ "),

$$
\begin{gathered}
G_{11} \simeq 1-2 N_{2}^{-1} \star\left[e^{2 \eta_{2}}+\frac{1}{\mathrm{v}}-\frac{1-\mathrm{v}}{\mathrm{v}} e^{\eta_{1}+\eta_{2}-i / 2 \lambda}\right] \star N_{2}^{1 / 2} \star D^{-1} \star N_{2}^{1 / 2} \\
-2 N_{1}^{-1} \star\left[e^{2 \eta_{1}}-\frac{1}{\mathrm{v}}+\frac{1+\mathrm{v}}{\mathrm{v}} e^{\eta_{1}+\eta_{2}+i / 2 \lambda}\right] \star N_{1}^{1 / 2} \star D^{-1} \star N_{1}^{1 / 2}, \\
G_{12} \simeq 2 i N_{2}^{-1} \star\left[e^{2 \eta_{2}}+\frac{1}{\mathrm{v}}-\frac{1-\mathrm{v}}{\mathrm{v}} e^{\eta_{1}+\eta_{2}-i / 2 \lambda}\right] \star N_{2}^{1 / 2} \star D^{-1} \star N_{2}^{1 / 2} \star e^{\eta_{1}} \\
-2 i N_{1}^{-1} \star\left[e^{2 \eta_{1}}-\frac{1}{\mathrm{v}}+\frac{1+\mathrm{v}}{\mathrm{v}} e^{\eta_{1}+\eta_{2}+i / 2 \lambda}\right] \star N_{1}^{1 / 2} \star D^{-1} \star N_{1}^{1 / 2} \star e^{\eta_{2}},
\end{gathered}
$$




$$
\begin{aligned}
& G_{21} \simeq-2 i N_{2}^{-1} \star\left[e^{\eta_{1}}-\frac{1-\mathrm{v}}{\mathrm{v}} e^{\eta_{2}}+\frac{1}{\mathrm{v}} e^{\eta_{1}+2 \eta_{2}-i \lambda}\right] \star N_{2}^{1 / 2} \star D^{-1} \star N_{2}^{1 / 2} \\
& +2 i N_{1}^{-1} \star\left[e^{\eta_{2}}+\frac{1+\mathrm{v}}{\mathrm{v}} e^{\eta_{1}}-\frac{1}{\mathrm{v}} e^{2 \eta_{1}+\eta_{2}+i \lambda}\right] \star N_{1}^{1 / 2} \star D^{-1} \star N_{1}^{1 / 2} \\
& G_{22} \simeq 1-2 N_{2}^{-1} \star\left[e^{\eta_{1}}-\frac{1-\mathrm{v}}{\mathrm{v}} e^{\eta_{2}}+\frac{1}{\mathrm{v}} e^{\eta_{1}+2 \eta_{2}-i \lambda}\right] \star N_{2}^{1 / 2} \star D^{-1} \star N_{2}^{1 / 2} \star e^{\eta_{1}} \\
& -2 N_{1}^{-1} \star\left[e^{\eta_{2}}+\frac{1+\mathrm{v}}{\mathrm{v}} e^{\eta_{1}}-\frac{1}{\mathrm{v}} e^{2 \eta_{1}+\eta_{2}+i \lambda}\right] \star N_{1}^{1 / 2} \star D^{-1} \star N_{1}^{1 / 2} \star e^{\eta_{2}} .
\end{aligned}
$$

There is some pattern with respect to the interchange $\eta_{1} \leftrightarrow \eta_{2}$ and regarding sign flips of $\mathrm{v}$ and $\lambda$, but no obvious symmetry under $\theta \rightarrow-\theta$. We have chosen the positions of the $N_{k}$ such that their arguments are not shifted. By construction (2.9), we have the equalities $G_{11}=G_{22}$ and $G_{12}=G_{21}$. These are not manifested in (5.6)-(5.9) because the neglected $O\left(\lambda^{2}\right)$ terms are not equal, but they are straightforward to verify at $O(\lambda)$. Note that, for the exact result, $D$ is to be inverted with respect to star multiplication. However, since $D_{\star}^{-1}-D^{-1}=O\left(\lambda^{2}\right)$, we may take the ordinary inverse in (5.6)-(5.9). Finally, the commutative limit collapses $G$ to (4.4), since all $N_{k}$ factors cancel and disappear.

We can also employ the last equation of (3.3), which expresses $G$ in terms of projectors only. After rescaling the projectors to

$$
\widetilde{P}_{1}=\frac{1-\mathrm{v}}{\mathrm{v}} P_{1} \text { and } \widetilde{P}_{2}=-\frac{1+\mathrm{v}}{\mathrm{v}} P_{2} \quad \text { such that } \widetilde{P}_{1} \star \widetilde{P}_{2}=\sigma P_{1} \star P_{2} \text {, }
$$

we rewrite

$$
\begin{aligned}
& G=1-2 P_{1} \star\left[1-\tilde{P}_{2} \star \tilde{P}_{1}\right]_{\star}^{-1} \star\left[1+\widetilde{P}_{2}\right]-2 P_{2} \star\left[1-\tilde{P}_{1} \star \widetilde{P}_{2}\right]_{\star}^{-1} \star\left[1+\widetilde{P}_{1}\right] \\
& =1-\frac{2 \mathrm{v}}{1-\mathrm{v}}\left[\tilde{P}_{1}+\widetilde{P}_{1} \star \widetilde{P}_{2}+\widetilde{P}_{1} \star \widetilde{P}_{2} \star \widetilde{P}_{1}+\cdots\right]+\frac{2 \mathrm{v}}{1+\mathrm{v}}\left[\tilde{P}_{2}+\widetilde{P}_{2} \star \widetilde{P}_{1}+\widetilde{P}_{2} \star \tilde{P}_{1} \star \tilde{P}_{2}+\cdots\right]
\end{aligned}
$$

In the last line, we have traded the notorious star inverses for formal geometric series,

$$
\left[1-\widetilde{P}_{j} \star \widetilde{P}_{k}\right]_{\star}^{-1}=\sum_{n=0}^{\infty}\left[\widetilde{P}_{j} \star \widetilde{P}_{k}\right]_{\star}^{n} \quad \text { with }(j, k)=(1,2) \quad \text { or } \quad(2,1)
$$

which may be truncated in an approximation for large velocities $\mathrm{v} \rightarrow 1$. In this way, $G$ is given as a power series in words $P_{j} \star P_{k} \star P_{j} \star \cdots \star P_{\ell}$. Remembering (3.4) and abbreviating also

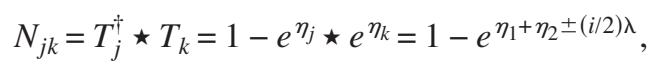

the "projector words" simplify to

$$
\begin{aligned}
P_{j} \star P_{k} \star P_{j} \star \cdots \star P_{\ell} & =\left(\begin{array}{c}
1 \\
\pm i e^{\eta_{j}}
\end{array}\right) N_{j}^{-1} \star N_{j k} \star N_{k}^{-1} \star N_{k j} \star \cdots \star N_{\ell}^{-1}\left(1, \pm i e^{\eta_{\ell}}\right) \\
& =\left(\begin{array}{c}
e^{-(1 / 2) \eta_{j}} \\
\pm i e^{(1 / 2) \eta_{j}}
\end{array}\right) \tilde{N}_{j}^{-1} \star \tilde{N}_{j k} \star \tilde{N}_{k}^{-1} \star \tilde{N}_{k j} \star \cdots \star \tilde{N}_{\ell}^{-1}\left(e^{-(1 / 2) \eta_{\ell}}, \pm i e^{(1 / 2) \eta_{\ell}}\right),
\end{aligned}
$$

where the last line is a symmetric rewriting with 


$$
\begin{aligned}
& \tilde{N}_{k}=e^{-\eta_{k}}+e^{\eta_{k}} \quad \text { and }
\end{aligned}
$$

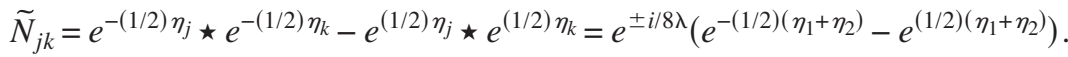

Pulling all together, one arrives at

$$
\begin{aligned}
& G_{11}=1-2 N_{1}^{-1}-2 N_{2}^{-1}+2 \frac{1+\mathrm{v}}{\mathrm{v}} N_{1}^{-1} \star N_{12} \star N_{2}^{-1}-2 \frac{1-\mathrm{v}}{\mathrm{v}} N_{2}^{-1} \star N_{21} \star N_{1}^{-1}+\cdots, \\
& G_{12}=2 i N_{1}^{-1} e^{\eta_{1}}-2 i N_{2}^{-1} e^{\eta_{2}}+2 i \frac{1+\mathrm{v}}{\mathrm{v}} N_{1}^{-1} \star N_{12} \star N_{2}^{-1} e^{\eta_{2}}+2 i \frac{1-\mathrm{v}}{\mathrm{v}} N_{2}^{-1} \star N_{21} \star N_{1}^{-1} e^{\eta_{1}}+\cdots, \\
& G_{21}=-2 i e^{\eta_{1}} N_{1}^{-1}+2 i e^{\eta_{2}} N_{2}^{-1}+2 i \frac{1+\mathrm{v}}{\mathrm{v}} e^{\eta_{1}} N_{1}^{-1} \star N_{12} \star N_{2}^{-1}+2 i \frac{1-\mathrm{v}}{\mathrm{v}} e^{\eta_{2}} N_{2}^{-1} \star N_{21} \star N_{1}^{-1}+\cdots \\
& G_{22}=1-2 e^{\eta_{1}} N_{1}^{-1} e^{\eta_{1}}-2 e^{\eta_{2}} N_{2}^{-1} e^{\eta_{2}}-2 \frac{1+\mathrm{v}}{\mathrm{v}} e^{\eta_{1}} N_{1}^{-1} \star N_{12} \star N_{2}^{-1} e^{\eta_{2}}+2 \frac{1-\mathrm{v}}{\mathrm{v}} e^{\eta_{2}} N_{2}^{-1} \star N_{21} \star N_{1}^{-1} e^{\eta_{1}} \\
& +\ldots,
\end{aligned}
$$

with $N_{k}$ and $N_{j k}$ to be taken from (5.5) and (5.13), respectively. This is an exact result. No star inverse needs to be taken, but we are left with infinite series, which may be summed in closed form only for $\theta=0$.

\section{EXPANDING IN $\theta$}

The task is to extract the deformed breather configuration

$$
e_{\star}^{ \pm \frac{i}{2} \phi_{ \pm}}=g_{ \pm}=G_{11} \pm G_{21}=G_{11} \pm G_{12}=: G_{e} \pm G_{o}
$$

from (5.6)-(5.9) or from (5.16), at least to subleading order in a $\theta$ expansion,

$$
f=f^{(0)}+\lambda f^{(1)}+\lambda^{2} f^{(2)}+\cdots \simeq f^{(0)}+\lambda f^{(1)} \text { for } f \in\left\{G, g_{ \pm}, \phi_{ \pm}, \cdots\right\} .
$$

Keeping $\mathrm{v}$ fixed and noticing that $e_{\star}^{h}=e^{h}+O\left(\lambda^{2}\right)$ for any function $h$, we have

$$
\phi_{ \pm} \simeq \mp 2 i \ln g_{ \pm} \simeq \mp 2 i \ln \left(g_{ \pm}^{(0)}+\lambda g_{ \pm}^{(1)}\right) \simeq \mp 2 i \ln g_{ \pm}^{(0)} \mp 2 i \lambda g_{ \pm}^{(1)} / g_{ \pm}^{(0)}=\phi_{ \pm}^{(0)} \mp 2 i \lambda g_{ \pm}^{(1)} e^{\mp \frac{i}{2} \phi_{ \pm}^{(0)}}
$$

and thus

$$
\begin{aligned}
& \frac{1}{2}\left(\phi_{+}+\phi_{-}\right) \simeq \varphi+i \lambda g_{+}^{(1)} e^{-\frac{i}{2} \varphi}-i \lambda g_{-}^{(1)} e^{\frac{i}{2} \varphi}=\varphi+2 \lambda G_{e}^{(1)} \sin \frac{\varphi}{2}+2 i \lambda G_{o}^{(1)} \cos \frac{\varphi}{2}, \\
& \frac{1}{2}\left(\phi_{+}-\phi_{-}\right) \simeq 2 \pi+i \lambda g_{+}^{(1)} e^{-\frac{i}{2} \varphi}+i \lambda g_{-}^{(1)} e^{\frac{i}{2} \varphi}=2 \pi+2 i \lambda G_{e}^{(1)} \cos \frac{\varphi}{2}+2 \lambda G_{o}^{(1)} \sin \frac{\varphi}{2}
\end{aligned}
$$

since $\phi_{+}^{(0)}=\varphi \pm 2 \pi$. From (5.16) one learns that, in the $\lambda$ expansion, the even orders of $G_{e}$ and the odd orders of $G_{o}$ are real while the odd orders of $G_{e}$ and the even orders of $G_{o}$ are imaginary. Because (6.4) must be real equations for $G \in \mathrm{U}(1) \times \mathrm{U}(1)$, this implies that 


$$
G_{e}^{(1)} \sin \frac{\varphi}{2}+i G_{o}^{(1)} \cos \frac{\varphi}{2}=0 \Rightarrow \phi_{+}^{(1)}=-\phi_{-}^{(1)}=2 i G_{e}^{(1)} / \cos \frac{\varphi}{2}=2 G_{o}^{(1)} / \sin \frac{\varphi}{2}=: 2 \chi,
$$

and so the sine-Gordon field $\frac{1}{2}\left(\phi_{+}+\phi_{-}\right)$gets deformed only at $O\left(\lambda^{2}\right)$ while the orthogonal combination $\frac{1}{2}\left(\phi_{+}-\phi_{-}\right)$is turned on at $O(\lambda)$. Interestingly, the relation (6.5) is again the commutative one, thus

$$
G \equiv\left(\begin{array}{cc}
G_{e} & G_{o} \\
G_{o} & G_{e}
\end{array}\right) \simeq e^{i \pi+i \lambda \chi}\left(\begin{array}{cc}
\cos \frac{\varphi}{2} & i \sin \frac{\varphi}{2} \\
i \sin \frac{\varphi}{2} & \cos \frac{\varphi}{2}
\end{array}\right) .
$$

For computing $\chi$ it suffices to look at any one of the $G$ matrix elements.

In order to expand $G$ to $O(\lambda)$ we need the first subleading term in multiple star products,

$$
f_{1} \star f_{2} \star f_{3} \star \cdots \star f_{n} \simeq f_{1} f_{2} f_{3} \cdots f_{n}+\frac{i}{2} \lambda \sum_{i<j} f_{1} \cdots\left(\partial_{[1} f_{i}\right) \cdots\left(\partial_{2} f_{j}\right) \cdots f_{n},
$$

where $\left(\partial_{[1} f_{i}\right)\left(\partial_{2} f_{j}\right) \equiv\left(\partial f_{i} / \partial \eta_{1}\right)\left(\partial f_{j} / \partial \eta_{2}\right)-\left(\partial f_{i} / \partial \eta_{2}\right)\left(\partial f_{j} / \partial \eta_{1}\right)$. The products appearing in (5.6)-(5.9) take the forms

$$
\begin{aligned}
& N_{2}^{-1} \star e^{h} \star N_{2}^{(1 / 2)} \star D^{-1} \star N_{2}^{(1 / 2)} \simeq e^{h} D^{-1}+\frac{i}{2} \lambda e^{h} D^{-2}\left(2 D \partial_{1} h \frac{N_{2}^{\prime}}{N_{2}}-\partial_{1} D \frac{N_{2}^{\prime}}{N_{2}}-\partial_{[1} h \partial_{2]} D\right), \\
& N_{1}^{-1} \star e^{h} \star N_{1}^{(1 / 2)} \star D^{-1} \star N_{1}^{(1 / 2)} \simeq e^{h} D^{-1}-\frac{i}{2} \lambda e^{h} D^{-2}\left(2 D \partial_{2} h \frac{N_{1}^{\prime}}{N_{1}}-\partial_{2} D \frac{N_{1}^{\prime}}{N_{1}}-\partial_{[2} h \partial_{1]} D\right), \\
& N_{2}^{-1} \star e^{h} \star N_{2}^{(1 / 2)} \star D^{-1} \star N_{2}^{(1 / 2)} \star e^{\eta_{1}} \\
& \simeq e^{h+\eta_{1}} D^{-1}+\frac{i}{2} \lambda e^{h+\eta_{1}} D^{-2}\left(2 D \partial_{1} h \frac{N_{2}^{\prime}}{N_{2}}-\partial_{1} D \frac{N_{2}^{\prime}}{N_{2}}-\partial_{[1} h \partial_{2]} D+\partial_{2} D-D \partial_{2} h\right), \\
& N_{1}^{-1} \star e^{h} \star N_{1}^{(1 / 2)} \star D^{-1} \star N_{1}^{(1 / 2)} \star e^{\eta_{2}} \\
& \simeq e^{h+\eta_{2}} D^{-1}-\frac{i}{2} \lambda e^{h+\eta_{2}} D^{-2}\left(2 D \partial_{2} h \frac{N_{1}^{\prime}}{N_{1}}-\partial_{2} D \frac{N_{1}^{\prime}}{N_{1}}-\partial_{[2} h \partial_{1]} D+\partial_{1} D-D \partial_{1} h\right),
\end{aligned}
$$

with $h$ being linear in $\eta_{1}$ and $\eta_{2}$. Collecting all terms and noticing cancellations we obtain

$$
\begin{gathered}
i v D^{2} G_{11}^{(1)}=-\partial_{1} D \frac{N_{2}^{\prime}}{N_{2}}-e^{\eta_{1}+\eta_{2}}\left(\partial_{1} D\left(1-\frac{N_{2}^{\prime}}{N_{2}}\right)-\partial_{2} D-D\left(1-2 \frac{N_{2}^{\prime}}{N_{2}}\right)\right)+(1 \leftrightarrow 2), \\
-D^{2} G_{12}^{(1)}=e^{\eta_{1}+2 \eta_{2}}\left(\partial_{1} D\left(2-\frac{N_{2}^{\prime}}{N_{2}}\right)+\partial_{2} D-2 D\right)+e^{2 \eta_{1}+\eta_{2}}\left(\partial_{1} D-2 D\right)\left(1-\frac{N_{2}^{\prime}}{N_{2}}\right)+(1 \leftrightarrow 2), \\
D^{2} G_{21}^{(1)}=e^{\eta_{1}}\left(\left(2 D-\partial_{1} D\right) \frac{N_{2}^{\prime}}{N_{2}}-\partial_{2} D\right)+e^{\eta_{2}} \partial_{1} D\left(1-\frac{N_{2}^{\prime}}{N_{2}}\right)+(1 \leftrightarrow 2),
\end{gathered}
$$




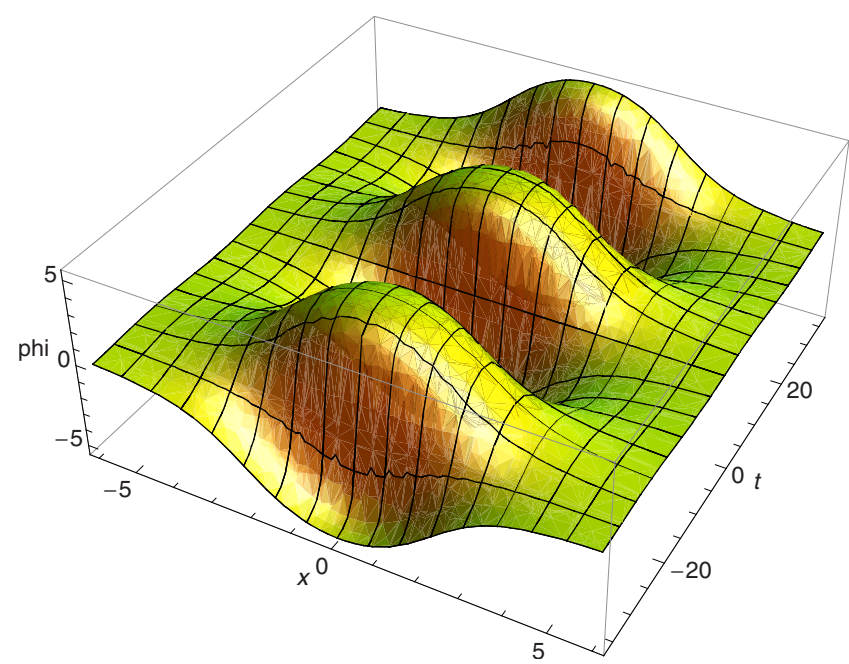

FIG. 1. (Color online) Commutative breather $\bar{\varphi}(t, x)$ for $\mathrm{v}=0.21$.

$$
i v D^{2} G_{22}^{(1)}=e^{2 \eta_{1}+2 \eta_{2}}\left(\partial_{1} D-2 D\right)\left(2-\frac{N_{2}^{\prime}}{N_{2}}\right)-e^{\eta_{1}+\eta_{2}}\left(\partial_{1} D\left(1-\frac{N_{2}^{\prime}}{N_{2}}\right)+\partial_{2} D-D\right)+(1 \leftrightarrow 2),
$$

which further collapses to

$$
\begin{gathered}
G_{e}^{(1)}=-2 i \mathrm{v} e^{\eta_{1}+\eta_{2}} \frac{\left(1-e^{\eta_{1}+\eta_{2}}\right)^{2}-\mathrm{v}^{2}\left(e^{\eta_{1}}+e^{\eta_{2}}\right)}{\left[\left(1-e^{\eta_{1}+\eta_{2}}\right)^{2}+\mathrm{v}^{2}\left(e^{\eta_{1}}+e^{\eta_{2}}\right)\right]^{2}}=-\frac{i \mathrm{v}}{2} \frac{\sinh ^{2} \gamma \mathrm{v} t-\mathrm{v}^{2} \cosh ^{2} \gamma x}{\left[\sinh ^{2} \gamma \mathrm{v} t+\mathrm{v}^{2} \cosh ^{2} \gamma x\right]^{2}}, \\
G_{o}^{(1)}=4 \mathrm{v}^{2} e^{\eta_{1}+\eta_{2}} \frac{\left(e^{\eta_{1}}+e^{\eta_{2}}\right)\left(1-e^{\eta_{1}+\eta_{2}}\right)}{\left[\left(1-e^{\eta_{1}+\eta_{2}}\right)^{2}+\mathrm{v}^{2}\left(e^{\eta_{1}}+e^{\eta_{2}}\right)\right]^{2}}=\mathrm{v}^{2} \frac{\sinh \gamma \mathrm{v} t \cosh \gamma x}{\left[\sinh ^{2} \gamma \mathrm{v} t+\mathrm{v}^{2} \cosh ^{2} \gamma x\right]^{2}} .
\end{gathered}
$$

Comparing to (4.4), we indeed confirm that $G^{(1)}=i \chi G^{(0)}$, and hence

$$
g_{ \pm} \simeq e^{i \pi+i \lambda \chi} e^{ \pm \frac{i}{2} \varphi} \quad \text { with } \chi=\frac{-\mathrm{v} / 2}{\sinh ^{2} \gamma \mathrm{v} t+\mathrm{v}^{2} \cosh ^{2} \gamma x} .
$$

It appears as if the sine-Gordon field gets deformed via $\varphi \rightarrow \varphi \mp 2 \lambda \chi$, but this is misleading.

This formula provides the explicit $O(\theta)$ correction to the commutative kink-antikink configuration. To obtain the breather, we still must analytically continue $\mathrm{v} \rightarrow i \mathrm{v}$, which yields ${ }^{2}$

$$
\lambda \rightarrow 2 \theta \frac{i \mathrm{v}}{1+\mathrm{v}^{2}}=: i \bar{\lambda} \quad \text { and } \quad \chi \rightarrow i \frac{\mathrm{v} / 2}{\sin ^{2} \bar{\gamma} \mathrm{v} t+\mathrm{v}^{2} \cosh ^{2} \bar{\gamma} x}=: i \bar{\chi},
$$

so that the leading correction to $G$ remains a phase factor. This is the main result of this letter. Clearly, $\chi$ oscillates with twice the classical breather frequency $\omega=\bar{\gamma} \mathrm{v}$. More generally, our construction shows that the deformed breather frequency does not depend on $\theta$ at all. In Figs. 1 and 2 we illustrate the shapes of $\bar{\varphi}(t, x)$ and $\bar{\chi}(t, x)$ for a typical value of $\mathrm{v}$.

\footnotetext{
${ }^{2}$ The comoving coordinates (3.1) turn complex and lose their physical interpretation. However, the fundamental Heisenberg algebra (5.1) of the actual $(t, x)$ coordinates and thus the nature of the deformation remains unchanged.
} 


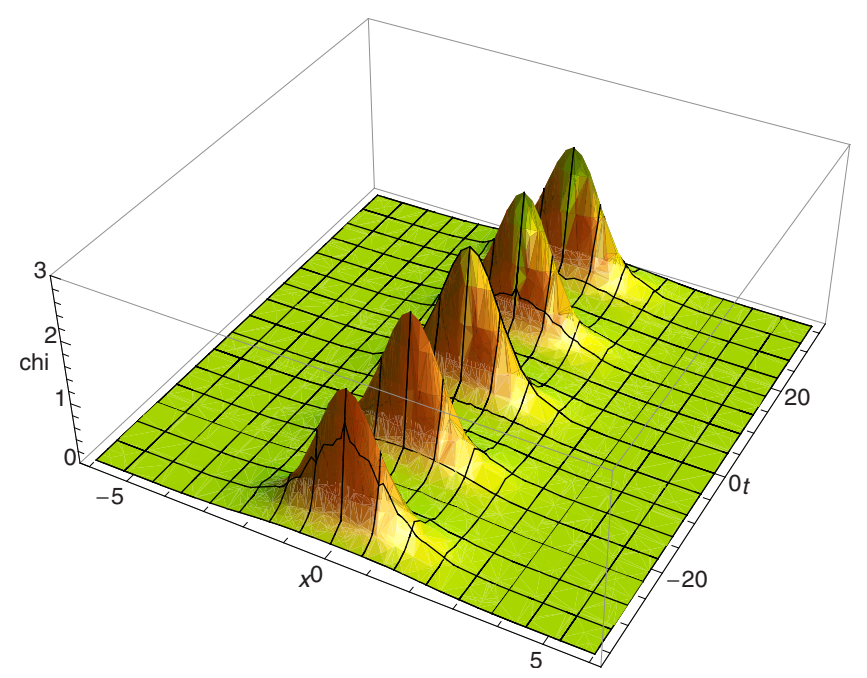

FIG. 2. (Color online) Noncommutative correction $\bar{\chi}(t, x)$ for $\mathrm{v}=0.21$.

\section{ACKNOWLEDGMENTS}

The authors are grateful to D. Harland, S. Kürkçüoğlu, and A. D. Popov for fruitful discussions. The work of A.F. and O.L. is partially supported by the Deutsche Forschungsgemeinschaft (Grant No. LE-838/9).

${ }^{1}$ R. Rajaraman, Solutions and Instantons. An Introduction to Solitons and Instantons in Quantum Field Theory (NorthHolland, Amsterdam, Netherlands, 1982).

${ }^{2}$ R. S. Ward, Philos. Trans. R. Soc. London, Ser. A 315, 451 (1985); L. J. Mason and G. A. J. Sparling, Phys. Lett. A 137, 29 (1989); J. Geom. Phys. 8, 243 (1992); M. J. Ablowitz, S. Chakravarty, and L. A. Takhtajan, Commun. Math. Phys. 158, 289 (1993); T. A. Ivanova and A. D. Popov, Phys. Lett. A 205, 158 (1995); Theor. Math. Phys. 102, 280 (1995); Teor. Mat. Fiz. 102, 384 (1995)]; S. Chakravarty, S. L. Kent, and E. T. Newman, J. Math. Phys. 36, 763 (1995); M. Legare, Int. J. Mod. Phys. A 12, 219 (1997).

${ }^{3}$ V. P. Nair and J. Schiff, Nucl. Phys. B 371, 329 (1992).

${ }^{4}$ R. S. Ward, J. Math. Phys. 29, 386 (1988); Commun. Math. Phys. 128, 319 (1990).

${ }^{5}$ O. Lechtenfeld and A. D. Popov, JHEP 11, 040 (2001); Phys. Lett. B 523, 178 (2001).

${ }^{6}$ O. Lechtenfeld, Fortschr. Phys. 52, 596 (2004).

${ }^{7}$ C. S. Chu and O. Lechtenfeld, Phys. Lett. B 625, 145 (2005).

${ }^{8}$ O. Lechtenfeld, L. Mazzanti, S. Penati, A. D. Popov, and L. Tamassia, Nucl. Phys. B 705, 477 (2005).

${ }^{9}$ S. Kürkçüoğlu and O. Lechtenfeld, JHEP 09, 020 (2007). 